\title{
A Collaborative Filtering Recommender System Model for Recommending Intervention to Improve Elderly Well-being
}

\author{
Aini Khairani Azmi ${ }^{1}$, Noraswaliza Abdullah ${ }^{2}$, Nurul Akmar Emran ${ }^{3}$ \\ Faculty of Information and Communication Technology \\ Universiti Teknikal Malaysia Melaka Hang Tuah Jaya \\ 76100 Durian, Tunggal, Melaka, Malaysia
}

\begin{abstract}
In improving elderly well-being nowadays, people at home or health care centre are mostly focusing on guarding and monitoring the elderly using tools, such as CCTV, robots, and other appliances that require a great deal of cost and neat fixtures to prevent damage. Elderly observations using the recommender system are found to be implemented, but only focusing on one aspect such as nutrition and health. However, it is important to give interventions to an elderly by concentrating more on the multiple aspects of successful ageing such as social, environment, health, physical, mental and other so that it can help the elderly people in achieving successful ageing as well as improving their well-being. In this paper, two recommender system models are proposed to recommend interventions for improving elderly well-being in the multiple aspects of successful ageing. These models using a Collaborative Filtering (CF) technique to recommend interventions to an elderly based on the interventions given to other elderly who have similar conditions with the user. The process of recommending interventions involves the generation of user profiles presenting the elderly conditions in multiple aspects of successful ageing. It also applying the k-Nearest Neighbor (kNN) method to find users with similar conditions and recommending interventions based on the interventions given to the similar user. The experiment is conducted to determine the performance of the proposed Collaborative Filtering (CF) recommender system and Collaborative Filtering and Profile Matching (CFS) compared to the Basic Search (BS). The results of the experiment showed that Collaborative Filtering (CF) recommender system and Collaborative Filtering and Profile Matching (CFS) outperformed Basic Search (BS) in terms of precision, recall and F1 measure. This result showed that the proposed models are efficient to recommend interventions using elderly profiles based on many aspects of successful ageing.
\end{abstract}

Keywords-Collaborative filtering; elderly well-being; $k$-nearest neighbor; recommender system; successful ageing

\section{INTRODUCTION}

The significant increase in life expectancy at birth has been achieved over the last century. A combination of medical advances, escalating health and social care costs, and higher expectations for older age have brought international interest in promoting healthier elderly and achieving "successful" ageing. According to [1], successful ageing includes three main components which are; 1) low probability of disease and disease-related disability, 2) high cognitive and physical functional capacity, and 3) active engagement in social and productive activities. A missing component to this three-factor model of successful ageing is identified in [1] which is positive spirituality which then became the fourth factor proposed in [3] to strengthen the model. Some consider health and functioning in old age as a prerequisite when striving for successful ageing [2]. Nutrition and environmental factors are also needed for elderly to age well, as well as achieving successful ageing [4][6]. Spiritual nowadays become more important to elderly in order to improve the quality of their life [7]-[8]. Therefore, it can be concluded that in order to achieve successful ageing, there are many aspects that need to be focused on for improving elderly well-being such as socialization, health, cognitive, physical, environment, nutrition and spirituality.

Elderly observations using the recommender system are found to be implemented, but only focusing on one aspect such as nutrition and health. There are some research works on recommender systems to improve elderly well-being such as recommending food and monitoring users' nutritional state by using user nutritional profile. According to [1], [2] and [3], multiple aspects must be considered in order for the elderly people to achieve successful ageing, as well as to improve their well-being. It is important to consider all the aspects or factors that contribute to successful ageing such as socialization, health, cognitive, physical, nutrition, spiritual and environment [1]-[8]. Those that achieve successful ageing have good conditions in all these aspects with no chronic illness, high levels of resilience, low rates of depression, good social network and also high levels of life satisfaction into their golden years. The elderly are most likely to age successfully with continuous monitoring and providing interventions for all these aspects. Therefore, a recommender system to recommend interventions by considering multiple aspects of successful ageing is required in order to improve elderly well-being and to help them achieve successful ageing.

In this paper, a recommendation model for recommending intervention in multi aspects of successful ageing for elderly people has been suggested by using the collaborative filtering technique. This technique utilized the elderly profiles created from the results of assessments conducted on various aspects of successful ageing such as socialization, health, cognitive, physical, nutrition, spiritual and environment. The k-Nearest Neighbor $(\mathrm{kNN})$ algorithm was used for finding similar users or elderly based on the profiles of their conditions on these 
aspects. Finally, interventions were recommended for the elderly based on the interventions that have been given to other elderly who have the same conditions or profiles.

This paper comprises five sections, where Section II describes the review of previous studies on the interventions for elderly, and Section III presents the methodologies which discussed the proposed user profiling and recommendation approach. In Section IV, experiment and evaluation results are discussed and this paper is concluded in Section V.

\section{LITERATURE REVIEW}

\section{A. Related Work}

The recommender system exists in many types of domains such as health, e-Commerce, movies, elderly well-being and many others. Usually, the Collaborative Filtering (CF) approach is used to recommend items to the user based on items that have been highly rated by similar users. There are many researches on recommender systems that have been conducted in each domain especially for frequently purchased items such as books and movies. This is because a large amount of ratings data can be easily accumulated and used by the recommender system to recommend these items for a new user. For recommending interventions, a large amount of ratings data is not available to be used by recommendation approaches to predict user's preferences. Thus, methods that can learn users' profiles without the availability of user's ratings are needed to be utilized by the recommendation approach.

A recommendation approach does exist for recommending interventions for elderly. There are some researches done on recommendation techniques for other types of items or services such as for recommending a recommendation related to the health [9] and nutrition [10]-[14]. In [9], the main focus of this recommender system is to build a digital platform focusing on people with dementia problem which most of this problem having by elderly. This recommender system provides interventions to caregivers and people living with dementia.

There are other recommender systems related with nutrition aspect. The nutritional semantic recommender system recommends a healthy diet plan to the elderly following expert guidelines [10]. It retrieves reliable and complete nutritional information from expert sources and manages this information by providing it to the users in the form of recommendations. A food recommendation proposed by [12] provides a healthy plan of diet for the elderly following expert guidelines which recommend the correct amount of nutrition to an elderly. It retrieved complete and reliable nutritional information from expert sources, such as people (nutritionists and gerontologists) or computerized systems (information systems and nutritional databases from the World Health Organization and, Spanish Society of Parenteral and Enteral Nutrition) and users in the context of these recommendations are offered to manage this information.

Another proposed intervention recommendation approach was focused on a demographic recommender system for the elderly [15]. This recommender system focused on the demographic aspect to provide elderly with information about services of health, recreation, household, etc. The purpose of this study is to enable older people to live longer, determine their choices at home by providing useful information on comprehensive resources and providing personal information about available services in the surroundings of the user.

Lifestyle of the elderly was also being looked into which provide support in the context of recommendations to help users cope with typical issues of everyday life and contributing positively to their welfare [16]. The development of an Ambient Assisted Living (AAL) system called CARE that serves as a test-bed for user studies is focusing on recommendations and interventions. The two potential peer groups of users were recruited. Structured interview among a batch of 20 Greek seniors, and a batch of 27 German seniors, which were formed earlier were conducted. The seniors' lifestyle, medical needs, attitude towards AAL technologies were focused on, and more specifically, the desired functions and system configurations of a recommendation given by the CARE system. The interview's results were discussed and the primary CARE prototype that emerged as an add-on digital image frame that duplicated photo exposure with reserves and interventions has been drawn to enhance the lifestyle and welfare of seniors. Enhancing the lifestyle of elderly improves their social skills, thus making them happier when talking to others.

From this previous research works, most of the research works on recommending interventions for elderly are focusing on only one aspect of successful ageing such as health or nutrition. To the best of our knowledge, there is no recommender system model that integrates various aspects of successful ageing as well as recommends interventions based on the elderly conditions of these aspects. This shows that, most of elderly did not get enough quality of the life when they not get proper intervention from each of every aspect towards the successful of their age. To improve the well-being of elderly and help them to age successfully, many other aspects should be considered such as socialization, health, cognitive, physical, nutrition, spiritual and environment. Therefore, a recommendation system that can recommend interventions based on various aspects of successful ageing is proposed. This recommendation is based on elderly profiles generated from the assessment of these aspects. The next section will discuss the proposed approach in more details.

\section{PROPOSED APPROACH}

For this experiment, in recommending the interventions to a new user, the following processes have been followed in order to make sure that the experiment went well and the detailed explanation are discussed in the section below:

- User Profiling: Building user profiles by using the assessment results of 7 aspects of successful ageing for identifying the elderly conditions.

- Neighborhood Formation: Collaborative Filtering was applied for selecting $\mathrm{k}$ number of nearest neighbors, based on the comparison of the new user's profile with the existing users' profiles.

- Recommendations: Selecting interventions and recommending interventions to the new user. 


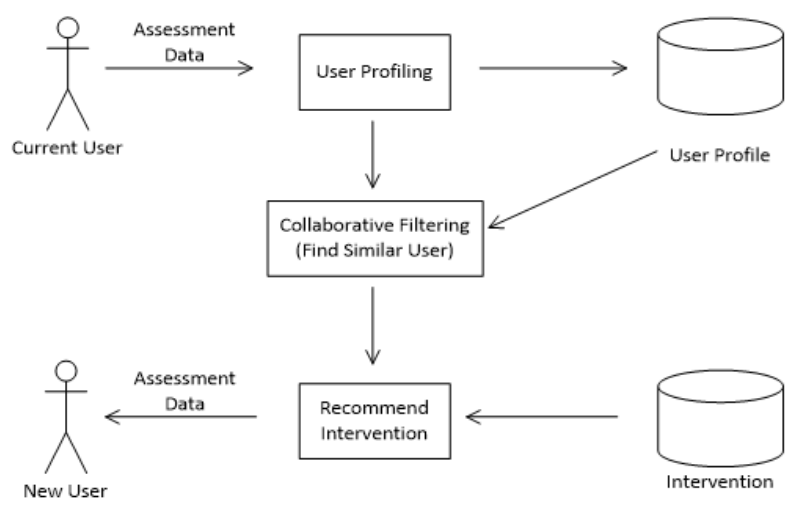

Fig. 1. A General Framework of the Interventions Recommendation.

Fig. 1 shows the framework for recommending interventions to the new users. A detailed explanation will be discussed in the section below.

\section{A. User Profiling}

User profiling is the process of gathering information about the topics or subjects in which a user is interested in. User profiles are the main source of information through which personalization systems can learn about users' interests or preferences. The accuracy and effectiveness of user profiling affects the performance of recommender systems. The crucial aspect of user profiles is their ability to represent users' current interests. In this research, the elderly assessment profiles are important and always being considered to recommend interventions that are suitable for their conditions. Therefore, the proposed recommender system must take the user assessment result for each aspect into consideration when making interventions recommendation for the elderly. The user assessment profile will be used by the proposed recommendation techniques to recommend interventions for improving elderly well-being.

User profiling process consists of two main phases. The first phase of user profiling is to collect information about the user. To be able to identify the needs of users, the recommender system needs to know something about the user. For this research, the first step in gathering information about elderly people was by collecting their assessment data to identify the level of their successful ageing. In order to measure the level of successful ageing, they went through a few assessments twice a year. There were 7 different aspects assessed for each elderly which are socialization, health, cognitive, physical, nutrition, spiritual and environment to determine their conditions. Therefore, we considered these 7 aspects in creating user profiles and intervention recommendations. From these assessments, the results were collected and used to construct user profiles.

The second phase in user profiling was profile construction and representation. An essential process of the personalized recommender systems is how to a build user profile, which represents the information needs and preference of a user and has great impact on the performance of recommendations. One important consideration when constructing a user profile is that more accurate user profiles need to be generated in order to provide more effective recommendations. For this step, each elderly will have his or her own user profile which consisted of 7 assessment results for each aspect. The user profiles were generated based on the information given by the experts from one of the elderly institution in Malaysia. Each aspect had different methods to calculate the assessment result in order to identify the elderly condition for the aspect. Then, the assessment result for each aspect was used to calculate the overall result based on the weightage given for each aspect. Finally, the user was classified into 3 different categories which are successful, semi-successful and non-successful based on the overall result of the assessments.

Basically, a user profile for elderly consisted of a vector of items and their ratings. User profile was used in suggesting an intervention for elderly. Below are the sample of user profiling for each user for this research.

$P_{i}=\left\{A_{i 1}, A_{i 2}, A_{i 3}, A_{i 4}, A_{i 5}, A_{i 6}, A_{i 7}\right\}$

where $\mathrm{A}$ is assessment aspect, $\mathrm{P}$ is user profile and $\mathrm{i}$ represents different users or elderly. There are 7 variables or aspects that present a user profile which are $A_{i 1}, A_{i 2}, A_{i 3}, A_{i 4}$, $A_{i 5}, A_{i 6}$ and $A_{i 7} . A_{i 1}$ represent the socialization aspect, $A_{i 2}$ represent the health aspect, $A_{i 3}$ represent the cognitive aspect, $A_{i 4}$ represent the physical aspect, $A_{i 5}$ represent the nutrition aspect, $A_{i 6}$ represent the spirituality aspect and $A_{i 7}$ represent the environment aspect for user i. The assessment method for each aspect was provided by the expert. The weightage was collected from the experts of each aspect in order to calculate the overall condition of the elderly. The weightages for each aspect are as follows: socialization (10), health (30), cognitive (15), physical (15), nutrition (10), spiritual (10) and environment (10). Below is the formula to calculate the weightage for each aspect based on the assessment data collected from the elderly.

\section{- Socialization}

A set of true and false questions $(\mathrm{T}=1 / \mathrm{F}=0)$ were given to elderly. They were given 15 questions in order to identify their social condition. The following formula was used to calculate the result for the elderly social condition.

$A_{i 1}=\frac{\sum Q S}{\sum T Q} \times 10 \%$

where $Q S$ is the total number of correct answers given by the elderly from the socialization assessment and $T Q$ is the total number of questions needed to be answered correctly.

- Health

There were 6 sections of the question consisting of 36 true and false questions $(\mathrm{T}=1 / \mathrm{F}=0)$ given to the elderly to answer in order to identify their health condition. The following formula was used to calculate the result for the elderly health condition.

$A_{i 2}=\frac{\sum Q H}{\sum T Q} \times 30 \%$

where $Q H$ is the total number of correct answers given by the elderly from the health assessment and $T Q$ is the total number of questions needed to be answered correctly. 


\section{- Cognitive}

There were 2 types of tests given to the elderly to answer which were the Mini Mental State Examination (MMSE) and Barthel test. MMSE test comprises of 11 sets of multiple choice questions while Barthel test comprises of 10 sets of multiple choice questions. The following formula was used to calculate the result for the elderly cognitive condition.

$A_{i 3}=\left(\frac{\sum Q C_{I}}{\sum T Q_{I}} \times 7.5 \%\right)+\left(\frac{\sum Q C_{2}}{\sum T Q_{2}} \times 7.5 \%\right)$

where $Q C_{1}$ is the total scores that have been answered by the elderly from the cognitive assessment (MMSE) and $T Q_{1}$ is the total number of questions needed to be answered correctly in the cognitive assessment (MMSE). Meanwhile, $Q C_{2}$ is the total scores that have been answered by the elderly from the cognitive assessment (Barthel Index) and $T Q_{2}$ is the total number of questions needed to be answered correctly in the cognitive assessment (Barthel Index).

\section{- Physical}

There were 2 types of tests conducted which were the Armcurl test and Time Up and Go (TUG) test. TUG test comprises of 12 true and false questions $(T=1 / F=0)$. However, an elderly was required to answer only one test according to their physical conditions. The following formula was used to calculate the result for the elderly physical condition.

$A_{i 4}=\left(\frac{\sum Q P_{I}}{\sum T Q_{I}} \times 15 \%\right)$ OR $A_{i 4}=\left(\frac{\sum Q P_{2}}{\sum T Q_{2}} \times 15 \%\right)$

where $Q P_{1}$ is the total scores that have been answered by the elderly from the physical assessment (Armcurl) and $T Q_{1}$ is the total number of questions needed to be answered correctly in the physical assessment (Armcurl). Meanwhile, $Q P_{2}$ is the total number of correct answers given by the elderly from the physical assessment (TUG) and $T Q_{2}$ is the total number of questions needed to be answered correctly in the physical assessment (TUG).

\section{- Nutrition}

There were 6 types of menus that the elderly must take in one day and the menu intake was checked daily by an assigned staff. Each menu taken by the elderly was given a score of 10 and for each menu not be taken by the elderly was deducted by 5. If an elderly has taken 6 menus in a day, he or she was given a full mark of 60 per day. For each session, the score was given based on 6 months per session. Each elderly was being assessed based on the menu taken for 6 months only per session.

$A_{i 5}=\frac{\sum Q N}{\sum T Q} \times 30 \%$

where $Q N$ is the total amount of menus taken by the elderly all day in one session (Session 1 is from January until June and Session 2 is from July until December) and $T Q$ is the total number of nutrition menus needed to be taken per session in the nutrition assessment.

\section{- Spiritual}

This aspect was divided into 2 sets of questions which were for Muslims and non-Muslims. 5 sections of questions for
Muslims elderly comprised 54 multiple choice questions whereas 4 sections of questions for non-Muslims elderly is comprised 31 multiple choice questions to be answered. The following formula was used to calculate the result for the elderly spiritual condition.

$A_{i 6}=\frac{\sum Q I}{\sum T Q} \times 10 \%$

where $Q I$ is the total number of correct answers given by the elderly from the spiritual assessment (Islam / Moral) and $T Q$ is the total number of questions needed to be answered correctly.

\section{- Environment}

There were 4 sections of questions given to the elderly to answer which included 25 questions. The following formula was used to calculate the result for the elderly environment condition.

$A_{i 7}=\frac{\sum Q E}{\sum T Q} \times 10 \%$

where $Q E$ is the total number of correct answers given by the elderly from the environment assessment and $T Q$ is the total number of questions needed to be answered correctly.

Finally, the overall result was calculated by summing up the results of all aspects and the elderly was categorized into successful, semi-successful and non-successful based on their overall result. The following formula was used to calculate the overall result.

$O i=\sum_{i=j}^{j} A_{i j}$

where $O$ is the overall result of assessment for each aspect taken by an elderly, while $i$ and $j$ are the aspects of successful ageing.

\section{B. Collaborative Filtering and k-Nearest Neighbor (kNN)}

The Collaborative Filtering $(\mathrm{CF})$ recommendation approach is the earliest approach used in the recommender system and also the most popular and widely implemented technique. Collaborative Filtering methods are based on the accumulation and analysis on large amounts of information about user behavior, activity or preferences and prediction of what users want based on their similarity to other users.

A key advantage of the Collaborative Filtering approach is that it does not rely on machine analyzable content and therefore it is capable of accurately recommending complex items such as movies without requiring an understanding of the item itself. Many algorithms have been used in measuring user similarity or item similarity in recommender systems. In this paper, the k-nearest neighbor $(\mathrm{kNN})$ approach and the cosine similarity were used in conducting the experiment. The key technique of $\mathrm{kNN}$ is to calculate the similarity between target user and the others, and then find the $\mathrm{k}$ nearest neighbors to predict the target user's interest or intervention.

The working $\mathrm{kNN}$ can be described, in the case of any unknown items as test items. The $\mathrm{kNN}$ classifier finds information from the training data that are almost similar; or almost close items as the test items. Among them, the $\mathrm{k}$ 
number of items that are closest to the test items is selected as the nearest neighbors of the test items. For this research, cosine similarity method was used to find the similarity. In this case, two users were regarded as two vectors in the $\mathrm{n}$ dimensional item space. The similarity between them was measured by computing the cosine of the angle between these two vectors. Formally, similarity between users A and B is given as below where $\mathrm{A}$ and $\mathrm{B}$ represent the $\mathrm{n}$ dimensional vectors those users accumulated on the $n$ items.

similarity $(A, B)=\frac{A \cdot B}{\|A\| \times\|B\|}=\frac{\sum_{i=1}^{n} A_{i} \times B_{i}}{\sqrt{\sum_{i=1}^{n} A_{i}^{2}} \times \sqrt{\sum_{i=1}^{n} B_{i}^{2}}}$

For this experiment, equation (10) was used in order to get the similarity value between two users. A represents the profile of existing user while B represents the profile of new user as discussed in section III. Each profile had 7 different values representing the result for each aspect. The Cosine Similarity values were calculated for other user profiles and the values were sorted to choose the selected (n) number of the most similar profiles. The number of $\mathrm{k}-\mathrm{NN}$ that has been chosen for this experiment was 5 because it achieved better results.

\section{Recommendation}

In this experiment, the interventions for each elderly profile were recommended based on the selected similar users. The profiles of the selected users were chosen based on the calculation of the 5 nearest neighbors. After the 5 neighbors were selected, the lists of all interventions of each neighbor were gathered accordingly. For the Basic Search (BS) approach, only interventions on the first nearest neighbor from 5 neighbors were selected to be recommended to the new active elderly, while for the Collaborative Filtering (CF) approach, all interventions from 5 nearest neighbor were ranked according to the frequency of interventions calculated from the intervention gathered from 5 neighbors. The highest number of interventions gathered for each aspect was selected to be recommended to the new or active elderly. For Collaborative Filtering with Profile Matching (CFS) approaches, the similarity of the user profile to the new user was calculated by matching each one of the 7 aspects of the nearest profile from 5 neighbors to the active or new one. The profile that matched or was nearest to the active or new profile was selected and the existing interventions given for that profile were selected to be recommended to the new or active one.

\section{EXPERIMENT}

This section focuses on the evaluation of the proposed user profiling and recommendation models. Firstly, the experiment design and the evaluation methods will be given. The experiments were conducted to see how the proposed user profiling and recommendation approaches performed by comparing them to the baseline approaches. Then, the results of experiments will be discussed and illustrated.

\section{A. Dataset}

A case study has been conducted for the elderly well-being domain. Data on the results of assessment for elderly was collected from one of the elderly institutions in Malaysia which is responsible for taking care of elderly people well-being. The data used in this research is an assessment data for seven (7) aspects of successful ageing collected from experts who conducted assessments to each elderly in each aspect of successful ageing. The intervention data were also collected from the interventions suggested by the experts. There were 139 samples of users involved in this research which were elderly aged above 60 living in this institution. The Collaborative Filtering recommender system prototype was also constructed to implement the interventions recommendations using user profiles constructed based on the assessment data. There were 139 user profiles created to be used by Collaborative Filtering; however, the user profiles can be accumulated for every assessment session which is conducted twice a year. The interventions were given to the elderly after each assessment session; therefore, the intervention data can also be collected for every assessment session.

There were 3 different recommendation approaches implemented in this experiment in order to compare the intervention recommendations given to an elderly by using each approach. The approaches were Basic Search (BS), Collaborative Filtering (CF) and Collaborative Filtering with Profile Matching (CFS). The BS approach represented the approach that is still widely used by the users which is to search based on the keyword they used. In this research, the keyword for the BS was represented by the value of each aspect in the user profile. The $\mathrm{CF}$ approach was used to recommend interventions based on the profiles of similar users which is by recommending frequent interventions given for the similar users. Meanwhile, in CFS, this approach recommended interventions based on the nearest profile to the active user profile which is by recommending interventions given to the user with the most similar profile for each aspect. The experiment results for these three approaches were compared to see their performance.

In this experiment, the 139 profiles of users were grouped into 3 which were A, B and C and each group had 46, 46 and 47 profiles respectively. Each run of experiment used one of the groups as the testing data and other groups as the training data. For the Basic Search (BS) approach, the profile of a user in the testing data was compared with other profiles in the training data and only interventions on the first nearest neighbor were selected to be recommended to the new active elderly. For CF and CFS approaches, the nearest 5 neighbors were chosen and the interventions gathered from these 5 neighbors will be selected and recommended to the new user. For the CF approach, the total number of each intervention was calculated and the highest number of interventions for each aspect was selected to be recommended to the new user. Meanwhile, for the CFS approach, the interventions were chosen and recommended to the new one by finding similar user profiles or the nearest user profile matching the 7 aspects from 5 neighbors to the active or new one. The results of these different approaches were then compared and evaluated using precision and recall to see whether the proposed approach performed better than other approaches. Further explanation on this method of evaluation is discussed in the next section. 


\section{B. Evaluation Metrics}

In this experiment, Precision, Recall and F1 Measurement metrics were used to evaluate the performance of the proposed models. Recall and Precision are the measures for measuring the efficiency and the suitability of the recommendation obtained by the recommender system.

Recall measures the ability of the system to present all the relevant items and it can be seen as the measure of completeness. Recall also refers to the percentages of the relevant recommendation that were retrieved, out of the total relevant recommendations. Precision measures the ability of the system to present only those items that are relevant, and it can be seen as the measure of exactness. It is also refers to the percentages of the relevant recommendation that were retrieved, out of the total retrieved recommendations.

Recall $=\frac{N M}{N T}$

Precision $=\frac{N M}{N R}$

where $N M$ is the number of suggested interventions matching the testing interventions, $N T$ is the number of interventions that should be returned and $N R$ is the number of returned suggested interventions. Finally, the average recall and precision for all profiles were calculated for each approach.

The F1 metric was used to provide a general overview of the overall performance.F1 metric was used to provide a general overview of the overall performance. The F1 measure combined the recall and precision results with an equal weight.

$f 1=\frac{2 \times \text { Precision } \times \text { Recall }}{\text { Precision }+ \text { Recall }}$

\section{Experimental Setup}

In order to evaluate the effectiveness of the proposed user profiling approach and the recommendation approaches, this paper implemented the proposed user profiling, recommendation approaches, and the baseline models. For this experiment, 139 profiles of users were used as testing and training datasets. There were three runs of experiment conducted and the 139 profiles were divided into 3 groups (A, $\mathrm{B}$ and $\mathrm{C}$ ). The division of dataset contained two (2) parts which were Training and Testing as illustrated in Fig. 2, 3 and 4.

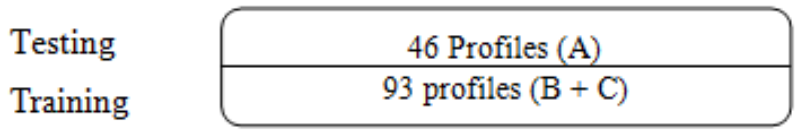

Fig. 2. The Division of Dataset for the Experiment (Run 1).

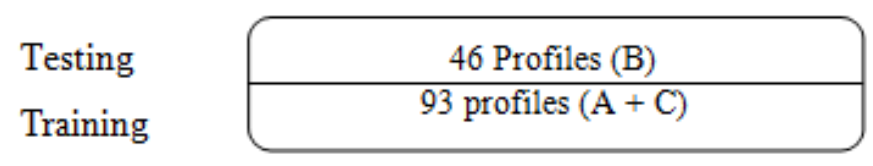

Fig. 3. The Division of Dataset for the Experiment (Run 2).

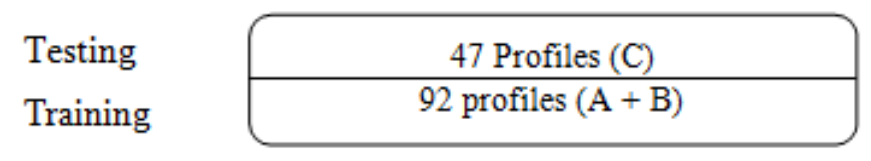

Fig. 4. The Division of Dataset for the Experiment (Run 3).
The testing dataset was considered as the target users or new users while the training dataset was considered as previous users. Training dataset was used to generate previous user's profiles which were used to find neighbors by using the neighborhood formation method. Finally, the average result for the 3 runs was calculated. The experiments were conducted to test if the proposed method was able to suggest interventions according to the current user's profile based on similar user profiles or neighbors. The interventions recommended for an elderly should improve the elderly well-being as the interventions were recommended based on the profiles of other elderly who have received the same interventions given by their caretakers.

\section{RESUlTS AND DISCUSSION}

Results of recommendations based on user profiles were examined and the results on Basic Search (BS), Collaborative Filtering (CF) and Collaborative Filtering with Profile Matching (CFS) approaches were compared. The objective of this set of experiments was to verify that the result of interventions by using Collaborative Filtering with Profile Matching (CFS) approaches can generate more accurate recommendations compared to $\mathrm{BS}$ approaches and $\mathrm{CF}$.

The table below shows the result of precision and recall for 3 different approaches. For these 3 approaches, the profile has been divided into 3 groups of runs and the results are been showed in the table. Table I shows the results of precision and recall for the BS approach. The results showed that precision for runs 1 and 3 was lower compared to recall at about 0.004 and 0.018 . For run 2, it showed that the precision got higher at 0.002 compared to recall. Table II shows the results of precision and recall for the $\mathrm{CF}$ approach. Each group of runs had higher precision results compared to recall about at 0.125 , 0.057 and 0.077. Meanwhile, Table III shows the results of precision and recall for the CFS approach and for this approach, the result was inverted with the $\mathrm{CF}$ approach in which the result of recall were more higher compare to precision at about $0.120,0.107$ and 0.091 . The differences in results of recall in this approach were much higher compared to the $\mathrm{CF}$ approach while the results of precision in this approach were much lower compared to the CF approach. Table IV shows the average results of precision and recall as well as the result for F1 measure for 3 experiment runs including basic search (BS), Collaborative Filtering (CF) and Collaborative Filtering with Profile Matching (CFS) based on different groups.

TABLE I. RESUlTS OF PRECISION AND RECALL (BS)

\begin{tabular}{|l|l|l|}
\hline Category & Precision & Recall \\
\hline Run 1 & 0.494 & 0.498 \\
\hline Run 2 & 0.455 & 0.453 \\
\hline Run 3 & 0.498 & 0.516 \\
\hline
\end{tabular}

TABLE II. RESULTS OF PRECISION AND RECALL (CF)

\begin{tabular}{|l|l|l|}
\hline Category & Precision & Recall \\
\hline Run 1 & 0.586 & 0.461 \\
\hline Run 2 & 0.606 & 0.549 \\
\hline Run 3 & 0.575 & 0.498 \\
\hline
\end{tabular}


TABLE III. RESULTS OF PRECISION AND RECALL (CFS)

\begin{tabular}{|l|l|l|}
\hline Category & Precision & Recall \\
\hline Run 1 & 0.508 & 0.628 \\
\hline Run 2 & 0.524 & 0.631 \\
\hline Run 3 & 0.506 & 0.597 \\
\hline
\end{tabular}

TABle IV. Average Results of Precision, ReCAll and F1 Measure FOR BS, CF, CFS APPROACHES

\begin{tabular}{|l|l|l|l|}
\hline Category & Precision & Recall & F1 Measure \\
\hline Basic search (BS) & 0.482 & 0.489 & 0.485 \\
\hline Collaborative Filtering (CF) & 0.589 & 0.503 & 0.543 \\
\hline $\begin{array}{l}\text { Collaborative Filtering and } \\
\text { Profile Matching (CFS) }\end{array}$ & 0.513 & 0.619 & 0.561 \\
\hline
\end{tabular}

From the results of these 3 different approaches shown in Table IV, it can be seen that BS approach has the lowest precision and recall values compared to the others. This is because the BS approaches only used the basic searching which only focused on the result gathered from only one neighbor who had the most match profile with the active user. Some of the recommended interventions were not matched with the active elderly profile. For CF approach, it can be seen that the precision is the higher compared to BS and CFS. The highest precision of $\mathrm{CF}$ shows that the interventions recommended by $\mathrm{CF}$ approach are accurate and suitable according to the new elderly profile or condition. The recall of CF is higher than BS but lower than CFS. This is because there are many other similar recommendations that can be given to an elderly but not necessarily recommended by the CF. Meanwhile, the result of CFS approach are inverted with the CF approach. CFS has the highest recall but lower precision than CF which is about 0.106. It shows that in term of recall, the CFS approach is able to recommend more interventions needed by the active elderly because the interventions recommended were based on the interventions of the aspects that were most similar to the active elderly aspect. However, in terms of precision, CFS is lower that CF. It shows that, not all recommended interventions matched with the interventions given to the previous user. This is because there were many similar interventions that can be given to an elderly for each aspect and the interventions were not just fixed to only interventions provided to the previous elderly, even though the interventions were also accurate.

In terms of F1 Measure, the results also show that CF performs better than the BS which is about 0.058. This is because for Basic Search (BS), it just considered interventions for only the most matching profile which led to inaccurate and not similar interventions being recommended. This shows that, when more similar profiles were being considered, the results were more precise and accurate. This is because many interventions can be recommended based on existing user profiles that may contribute to more accurate recommendations result of assessment or profile of user. However, the F1 Measure results show that CFS performs better than CF with 0.018 value difference. This is because the $\mathrm{CF}$ approach only took interventions that are most frequently given for each aspect among the 5 neighbors while the CFS approach considered the interventions for each aspect that had the most similar aspect value of the profiles among the 5 neighbors.

\section{CONCLUSION}

Problem of ageing has been recognized as a major social problem by sociologists all over the world. In order to ensure that the elderly continue to flourish, it is important to give some interventions to them following the multiple aspects of successful ageing which are social, health, physical, cognitive, nutrition, spiritual, nutrition and environment. We have proposed a recommender system for recommending suitable interventions to the elderly based on interventions given to other elderly who have similar profiles or assessment results. The results of the experiment showed that the more accurate recommendation of interventions can be provided to the new elderly by using the Collaborative Filtering approach. The results showed that $\mathrm{CF}$ and CFS performed better than BS in terms of precision, recall and F1 Measure. However, recall for $\mathrm{CF}$ approach was lower than CFS because CF recommended interventions based on frequent interventions suggested for some similar users. In term of precision, CF was the highest which showed that the recommended interventions by this approach were accurate and suitable for the new elderly with the same profile or condition. The CFS had higher recall than $\mathrm{CF}$ and lower precision that CF. However the CFS gave the best result for F1 Measure which showed that this approach was the best approach among the other. This is because CF approach only took interventions that were most frequently given for each aspect among the 5 neighbors while CFS approach considered the interventions for each aspect that had the most similar aspect value of the active elderly profiles among the 5 neighbors. Thus, CFS suggested interventions that were most suitable for each aspect of active elderly profile. To conclude, the $\mathrm{CF}$ and $\mathrm{CFS}$ were able to recommend interventions based on user profiles generated from the assessment results, therefore the recommended interventions may improve the elderly well-being in multiple aspects of successful ageing.

\section{LIMITATION}

There are a few limitations that can be identified throughout this experiment in term of data collections. Data has been collected from only 2 sessions of evaluation and intervention for each of elderly throughout the year. This data limitation may restrict the possible interventions that can be given to the new elderly. To address this limitation, we plan to propose a hybrid recommender system model by combining knowledge-based and collaborative filtering for recommending more accurate intervention for an elderly. A knowledge-based recommender system can be applied to recommend interventions based on the knowledge gathered from the domain expert. After the user profiles and interventions have been accumulated, the collaborative filtering approach can be used to recommend interventions for the new elderly based on the intervention given for other elderly who have similar profiles.

\section{ACKNOWLEDGMENT}

The authors would like to express their sincere appreciation for those who have participated in this research study. The authors of this paper are also appreciative to the Department of Information and Communication Technology at Universiti Teknikal Malaysia Melaka (UTeM) in supporting this work. 


\section{REFERENCES}

[1] Wahl, H., Deeg, D., \& Litwin, H., "Successful ageing as a persistent priority in ageing research," European Journal of Ageing, vol. 13, pp. 13, 2016.

[2] Cosco, T. D., K. Howse, and C. Brayne., "Healthy ageing, resilience and wellbeing," Epidemiology and Psychiatric Sciences, vol.26, no.6, pp. 579-583, 2017.

[3] Zimmer, Z., Jagger, C., Chiu, C. T., Ofstedal, M. B., Rojo, F., \& Saito, Y., "Spirituality, religiosity, aging and health in global perspective: A review," SSM-population health, vol.2, pp. 373-381, 2016.

[4] Aleksandrova, K., Pounis, G., \& di Giuseppe, R., "Diet, Healthy Aging, and Cognitive Function," In Analysis in Nutrition Research, pp. 321336, 2019.

[5] Costa, A., Carrión, S., Puig-Pey, M., Juárez, F., \& Clavé, P., "Triple Adaptation of the Mediterranean Diet: Design of A Meal Plan for Older People with Oropharyngeal Dysphagia Based on Home Cooking," Nutrients, vol.11, no.2, pp. 425, 2019.

[6] Oyeyemi, A. L., Kolo, S. M., Oyeyemi, A. Y., \& Omotara, B. A., "Neighborhood environmental factors are related to health-enhancing physical activity and walking among community dwelling older adults in Nigeria," Physiotherapy theory and practice, vol.35, no.3, pp. 288297, 2019.

[7] Gautam, S., Neville, S., \& Montayre, J. "What is known about the spirituality in older adults living in residential care facilities? An Integrative review" International journal of older people nursing, 2019.

[8] Ali, J., Marhemat, F., Sara, J., \& Hamid, H., "The relationship between spiritual well-being and quality of life among elderly people," Holistic nursing practice, vol.29, no.3, pp 128-135, 2015.
[9] Oliva-Felipe, Luis, et al. "Health Recommender System design in the context of CAREGIVERSPRO-MMD Project." Proceedings of the 11th PErvasive Technologies Related to Assistive Environments Conference. ACM,2018.

[10] V. Espín, M. V. Hurtado, and M. Noguera, "Nutrition for Elder Care: A nutritional semantic recommender system for the elderly," Expert Syst., vol. 33, no. 2, pp. 201-210, 2016.

[11] G. Agapito et al., "DIETOS: A recommender system for adaptive diet monitoring and personalized food suggestion," Int. Conf. Wirel. Mob. Comput. Netw. Commun., 2016.

[12] S. Bundasak and K. Chinnasarn, "EMenu recommender system using collaborative filtering and Slope One Predictor," Proc. 2013 10th Int. Jt. Conf. Comput. Sci. Softw. Eng. JCSSE 2013, pp. 37-42, 2013.

[13] V. Espín, M. V. Hurtado, and M. Noguera, "Nutrition for Elder Care: A nutritional semantic recommender system for the elderly," Expert Syst., vol. 33, no. 2, pp. 201-210, 2016.

[14] T. Rist, A. Seiderer, S. Hammer, M. Mayr, and E. André, "CARE Extending a Digital Picture Frame with a Recommender Mode to Enhance Well-Being of Elderly People," Proc. 9th Int. Conf. Pervasive Comput. Technol. Healthc., 2015.

[15] C. Stiller, F. Roß, and C. Ament, "Demographic recommendations for WEITBLICK, an assistance system for elderly," Isc. 2010 - 201010 th Int. Symp. Commun. Inf. Technol., pp. 406-411, 2010.

[16] S. Hammer et al., "Design of a lifestyle recommender system for the elderly: Requirement Gatherings in Germany and Greece," In Proceedings of the 8th ACM International Conference on PErvasive Technologies Related to Assistive Environments, pp. 80, 2015. 